\title{
N-Acetyl-D-Glucosamine Kinase Interacts with Dynein-Lis1-NudE1 Complex and Regulates Cell Division
}

\author{
Syeda Ridita Sharif ${ }^{1}$, Md. Ariful Islam ${ }^{1}$, and II Soo Moon ${ }^{1,2, *}$
}

\begin{abstract}
$N$-acetyl-D-glucosamine kinase (GIcNAc kinase or NAGK) primarily catalyzes phosphoryl transfer to GIcNAc during amino sugar metabolism. Recently, it was shown NAGK interacts with dynein light chain roadblock type 1 (DYNLRB1) and upregulates axo-dendritic growth, which is an enzyme activity-independent, non-canonical structural role. The authors examined the distributions of NAGK and NAGK-dynein complexes during the cell cycle in HEK293T cells. NAGK was expressed throughout different stages of cell division and immunocytochemistry (ICC) showed NAGK was localized at nuclear envelope, spindle microtubules (MTs), and kinetochores (KTs). A proximity ligation assay (PLA) for NAGK and DYNLRB1 revealed NAGK-dynein complex on nuclear envelopes in prophase cells and on chromosomes in metaphase cells. NAGKDYNLRB1 PLA followed by Lis1/NudE1 immunostaining showed NAGK-dynein complexes were colocalized with Lis1 and NudE1 signals, and PLA for NAGK-Lis1 showed similar signal patterns, suggesting a functional link between NAGK and dynein-Lis1 complex. Subsequently, NAGK-dynein complexes were found in KTs and on nuclear membranes where KTs were marked with CENP-B ICC and nuclear membrane with lamin ICC. Furthermore, knockdown of NAGK by small hairpin (sh) RNA was found to delay cell division. These results indicate that the NAGK-dynein interaction with the involvements of Lis1 and NudE1 plays an important role in prophase nuclear envelope breakdown (NEB) and metaphase MT-KT attachment during eukaryotic cell division.
\end{abstract}

\footnotetext{
${ }^{1}$ Department of Anatomy, ${ }^{2}$ Section of Neuroscience, Dongguk Medical Institute, Dongguk University Graduate School of Medicine, Gyeongju 38066, Korea

*Correspondence: moonis@dongguk.ac.kr
}

Received 3 May, 2016; revised 2 August, 2016; accepted 9 August, 2016; published online 20 September, 2016

Keywords: cell division, dynein, microtubule, kinetochore, Lis1, NAGK, nuclear envelope, NudE1

\section{INTRODUCTION}

$\mathrm{N}$-acetylglucosamine kinase (GIcNAc kinase or NAGK; E.C 2.7.1.59) was first discovered in 1970 (Datta, 1970), and it's mRNA and proteins were later found to be expressed in a variety of cell lines and tissues (Hinderlich et al., 2000, Ligos et al., 2002). NAGK belongs to the sugar kinase/heat shock protein 70/actin superfamily (Hurley, 1996), which is characterized by a $\mathrm{V}$-shaped fold consisting of two domains with the $\beta \beta \beta \alpha \beta \alpha \beta \alpha$ configuration. Members of this super family bind substrates and undergo a large conformational change involving a reduction of gap angle between these two domains (Hurley, 1996). The canonical function of NAGK is to phosphorylate GIcNAc to produce GlcNAc-6-phosphate in the GIcNAc recycling salvage pathway. The GlcNAc-6-phosphate is subsequently used to produce UDP-GICNAc that is utilized to synthesize $\mathrm{N}-/ \mathrm{O}$ glycans and glycolipids (Hakomori, 2000; Schachter, 2000; Van den Steen et al., 1998), and glycosaminoglycans (Esko and Lindahl, 2001).

In addition to its enzymatic role as a kinase, our laboratory reported a non-canonical, i.e., a non-enzymatic function for NAGK, which was first implicated in the differential distributions of the protein in different types of brain cells. In the mature rat brain, NAGK is present predominantly in neuronal dendrites and its levels are very low in neuronal axons and in the processes of neuroglial cells, such as, astrocytes and oligodendrocytes (Lee et al., 2014a), suggesting it has a specific role in neuronal dendrites. Furthermore, exogenous overexpression of NAGK promoted dendritic complexity in cultured rat hippocampal neurons, whereas its knockdown caused dendritic degeneration (Lee et al., 2014a). Surprisingly, the overexpressions of kinase-deficient mutant NAGKs also upregulated dendritic arborization and overexpression of the NAGK small domain, but not of the large domain, resulted in dendritic degeneration (Lee et al., 2014b), confirming a non-canonical structural function of NAGK. A similar non-canonical function associated with axonal arborization was observed in the developing axons of hippocampal neurons (Islam et al., 2015b). In order to elucidate the structural role of NAGK, Islam et al. (2015a) performed yeast two-hybrid screening and found NAGK interacted with dynein light chain roadblock type 1 (DYNLRB1). Interaction between NAGK and dynein molecular motor was further confirmed by immunocytochemistry (ICC) and proximity ligation assay (PLA). And by transfecting a peptide derived from the C-terminal DYNLRB1, which interacted with NAGK, interfered with NAGK 
function during neuronal development and resulted in dendrite degeneration and delayed axonal growth (Islam et al., 2015a; 2015b). Interestingly, NAGK-dynein interactions occur in the branch points of developing neuronal dendrites and axons where NAGK-dynein complexes are associated with Golgi outposts (Islam et al., 2015a, 2015b). Since these outposts serve as local microtubule-organizing centers (Ori-McKenney et al., 2012), evidence to date suggests that NAGK regulates dynein motor complex during the directional transport of Golgi outposts to branching sites, and/or during the microtubuleorganizing function of Golgi outposts in dendritic and axonal branch points.

In contrast to cytoplasmic expression, NAGK is ubiquitously present in nuclei. Yeast two-hybrid selections have shown that NAGK interacts with small nuclear ribonucleoprotein (snRNP)associated polypeptide $\mathrm{N}$ (snRNPN), and general transcription factor IIH polypeptide 5 (GTF2H5) and an ICC study showed the colocalization of NAGK signals with speckle and paraspeckle, suggesting its participation in gene expression (Sharif et al., 2015). Furthermore, NAGK ICC signals were reported to surround the cytoplasmic face of the outer nuclear membrane (Sharif et al., 2015). In our previous studies, we found NAGK interacts with cytoplasmic dynein motor complex that is also present on nuclear envelopes (Hu et al., 2013). Others have implicated dynein in prophase nuclear envelope breakdown (NEB) and mitotic chromosome separation based on its microtubule-anchoring activities during cell division (Sharp et al., 2000; Salina et al., 2002). Although the molecular mechanisms responsible for cell division have been substantially elucidated, the ways in which they are organized and controlled to create an effective, accurate cellular machine are yet to be explored. In the present study, we focused on the modulatory effect of NAGK on dynein function during different phases of cell division.

\section{MATERIALS AND METHODS}

\section{Antibodies and plasmids}

The following antibodies were used at the indicated dilutions: chicken polyclonal NAGK (1:1000 for ICC; GW22347, Sigma, USA); mouse monoclonal NAGK (1:10 for PLA; Santa Cruz Biotechnology, USA); rabbit polyclonal NAGK (1:50 for PLA; GeneTex, USA); rabbit polyclonal DYNLRB1/LC7 (1:50 for ICC and 1:25 for PLA; Proteintech Group, USA); mouse polyclonal dynein IC1 (1:25 for PLA; Santa Cruz Biotechnology); rabbit polyclonal Lis1 (Santa Cruz); rabbit polyclonal NudE1 (1:50 for ICC, Proteintech Group); rabbit polyclonal CENP-B (1:500; Abcam, UK); mouse monoclonal alpha-tubulin (1:10; broth preparation, Developmental Studies Hybridoma Bank, University of lowa, USA); rabbit polyclonal lamin B (1:1000; Young In Frontier Inc., Korea). The fluorescently labeled secondary antibodies Alexa Fluor 488 and 568 (Invitrogen, USA) were used to detect primary antibodies. The plasmids used for transfection were pDsRed2 vector and NAGK short hairpin (sh) RNA, as previously described (Lee et al., 2014a; 2014b).

\section{Cell culture, nocodazole treatment, and transfection} GT1-7 and HEK293T cells were grown on polylysine coated glass coverslips in DMEM (Invitrogen) supplemented with $10 \%$ fetal bovine serum and $1 \%$ Penicillin Streptomycin. Cells were treated with $70 \mathrm{ng} / \mathrm{ml}$ nocodazole (Sigma, USA) for $18.5 \mathrm{~h}$ in the same medium. HEK293T cells were transfected with indicated plasmids using Lipofectamine ${ }^{\circledR} 2000$ reagent (Invitrogen) according to the manufacturer's instructions.
Immunocytochemistry (ICC)

Cells were fixed by sequential paraformaldehyde/methanol fixation (Moon et al., 2007). Briefly, cells were incubated in 4\% paraformaldehyde in PBS (20 mM sodium phosphate buffer, $\mathrm{pH} 7.4,0.9 \% \mathrm{NaCl}$ ) at RT for $10 \mathrm{~min}$, and then incubated in methanol at $-20^{\circ} \mathrm{C}$ for $20 \mathrm{~min}$. Fixed cells were first incubated in preblocking buffer ( $5 \%$ normal goat serum, $0.05 \%$ Triton X-100 in PBS, pH 7.4) at $4^{\circ} \mathrm{C}$ for more than $6 \mathrm{~h}$, then treated with indicated antibodies, and mounted on glass slides using mounting medium containing DAPI.

\section{Proximity Ligation Assay (PLA)}

Generic in situ PLA was performed using a Duolink assay kit (Olink Bioscience, Sweden), according to the manufacturer's instructions with minor modifications. Briefly, cells were incubated with primary antibodies in preblocking buffer $(5 \%$ normal goat serum, $0.05 \%$ Triton $\mathrm{X}-100$ in PBS, $\mathrm{pH} 7.4$ ) at $4^{\circ} \mathrm{C}$ overnight, and then washed three times for 20 min in preblocking buffer at RT. For secondary antibodies conjugated with oligonucleotides, PLA probe anti-mouse MINUS and PLA probe antirabbit PLUS were diluted in preblocking buffer and applied for 2 $h$ at $37^{\circ} \mathrm{C}$ in a humidity chamber. Other aspects of the assay were performed according to the manufacturer's instructions. For the combination of PLAs and ICC, the PLA steps were performed first and then primary antibodies were added to cells overnight at $4^{\circ} \mathrm{C}$. Cells were then incubated with fluorophore conjugated secondary antibodies as per the above mentioned ICC procedure.

Image acquisition

An Olympus microscope BX53 equipped with UNA, BNA, and GW filter sets was used to take epifluorescence images. Images were acquired at high-resolution using a digital camera DP72 (Olympus, Japan) using CellSens Imaging Software (Olympus), and processed using Adobe Systems Photoshop 7.0 software (Adobe, USA).

\section{Statistics}

Cells $(n=500)$ from 2-3 different positions on each coverslip were counted at $48 \mathrm{~h}$ and $72 \mathrm{~h}$ after transfection and numbers of transfected cells were then expressed as percentages of all cells.

\section{RESULTS}

NAGK was expressed throughout the different stages of cell division

In interphase HEK293T cells, NAGK was present in cytoplasm, in nuclear speckles and paraspeckles, and on nuclear envelopes (Sharif et al., 2015). To study NAGK expression patterns during cell division we performed ICC using anti-NAGK and anti-tubulin antibodies in asynchronous HEK293T cells and followed by DAPI staining. NAGK expression in interphase cells was in line with our previous findings (Sharif et al., 2015). Cytoplasmic NAGK signals were mostly localized on microtubules (MTs) (Fig. 1A, Interphase, arrowheads) as was in previous observations in hippocampal neurons (Islam et al., 2015a). At prophase when chromosomes started to condense, some NAGK signals colocalized with MT on nuclear envelopes (Fig. 1B, Prophase, inset, arrowheads). During metaphase, condensed chromosomes are sequentially attached to spindle microtubules at their kinetochores, and during this stage, NAGK signals localized on thin MT spindle shafts and at the plus ends of spindles attached to chromosomes (Fig. 1C, Metaphase, 

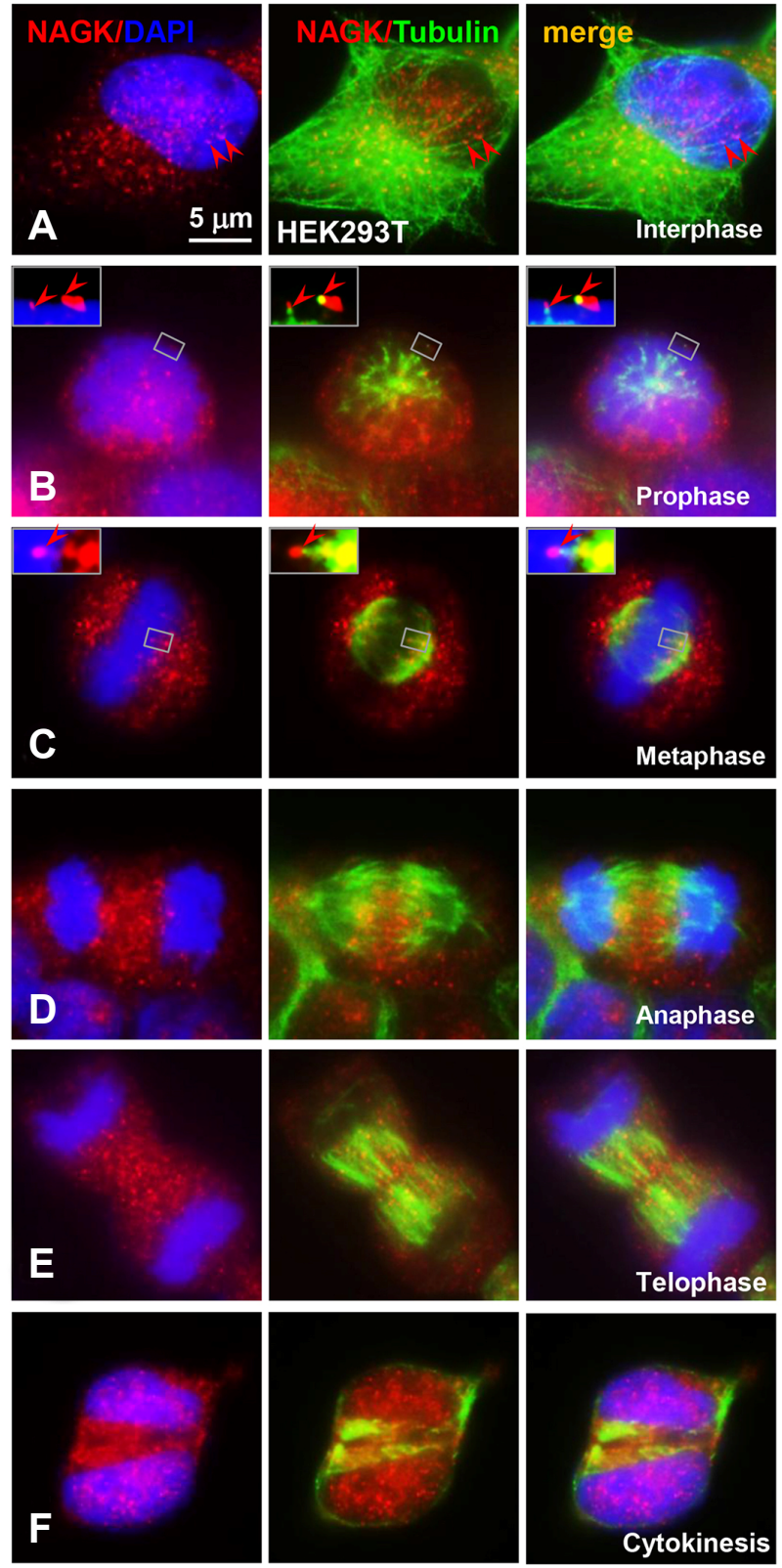

Fig. 1. NAGK expression profile during the cell cycle. Asynchronous HEK293T cells were fixed and double labeled with chicken anti-NAGK $(1: 1,000)$ and anti-tubulin (1:10) primary antibodies and then stained with DAPI. Primary antibodies were visualized using goat secondary antibodies conjugated to Alexa 488 (tubulin, green) or Alexa 568 (NAGK, red) fluorochromes. (A) NAGK distribution in interphase. Localization of NAGK puncta to MT fibers is indicated by arrowheads. (B) In prophase, colocalization of NAGK puncta with tubulin staining on nuclear envelope are shown by arrowheads (inset). (C) In metaphase, NAGK signals were observed on MTs, and cellular regions. Arrowheads show NAGK puncta at the tip of MT spindle fiber (inset). In anaphase (D) and telophase (E), similar NAGK localizations on MTs, chromosomes, and at other areas were observed, and during cytokinesis $(F)$, NAGK signals regained their characteristic interphase pattern and exhibited nuclear expression. Scale bar; $5 \mu \mathrm{m}$. inset, arrowheads). A similar distribution pattern was observed during anaphase (Fig. 1D, Anaphase) and telophase (Fig. 1E, Telophase). Finally, as the cytokinesis phase approached, NAGK started to redistribute throughout cells, including nuclei (Fig. 1F, Cytokinesis). These observations suggest that NAGK contributes to the anchoring of MT spindles to the nuclear envelope during prophase and to chromosomes during nucleokinesis.

NAGK interacted with dynein light chain roadblock type 1 (DYNLRB1) during cell division

Previously, we reported that NAGK interacted with DYNLRB1 subunits of dynein complex (Islam et al., 2015a). Dynein localizes to a variety of subcellular structures during G2 and mitosis, including the nuclear envelope (NE), centrosomes, kinetochores (KTs), mitotic spindles, and the cell cortex (Dujardin and Vallee, 2002; Kiyomitsu and Cheeseman, 2012; Pfarr et al., 1990; Steuer et al., 1990; Tanenbaum et al., 2010). In particular, dynein is involved in chromosome movements, spindle organization and positioning, and checkpoint silencing during mitosis (Howell et al., 2001; Sharp et al., 2000; Varma et al., 2008). Therefore, we investigated the in situ localization of NAGKdynein interactions through different stages of cell division by performing PLA on HEK293T cells for NAGK and DYNLRB1 and then followed with tubulin ICC and DAPI counter-staining (Fig. 2). PLA signals were observed at different $z$-axis layers of cells but were mostly studied at the equatorial nuclear layer. In interphase cells, NAGK-DYNLRB1 interactions (PLA assay) were found in cytoplasmic areas with many PLA signals on and around the nuclear envelope (Fig. 2A, arrowheads). We also observed NAGK-dynein interactions on the prophase nuclear envelope when chromosomes began to condensate (Fig. 2B, arrowheads). Dynein plays a critical role in nuclear envelope invagination during early prophase (Salina et al., 2002), and thus, NAGK-DYNLRB1 interactions may be a part of the dynein-adaptor protein complexes that guide prophase nuclear invagination prior to NEB. At metaphase, chromosomes aligned themselves at the equatorial plane and NAGK-dynein interactions (PLA) occurred on the mitotic spindle shafts and on the chromosomes (Fig. 2C, red arrowheads), and in peripheral cytoplasmic areas (Fig. 2C, white arrowheads). These PLA signals in peripheral cytoplasm may be on astral MTs, because dynein links these MTs to the cell cortex during cell division. Similar localizations of NAGK-DYNLRB1 PLA signals were observed during anaphase, telophase, and cytokinesis (Figs. $2 \mathrm{D}-2 \mathrm{~F}$, red arrowheads-on chromosomes and/or on polar MTs, white arrowheads-others). These observations indicate NAGK might regulate dynein function on mitotic MT spindles and at kinetochores (KTs) on chromosomes.

NAGK interacted with Lis1 during cell division on nuclear envelopes

Previous studies suggest dynein plays its role in cell division with the cooperation of Lis1 and NudE1/NudEL1 (Shu et al., 2004; Yan et al., 2003). Raaijmakers et al. (2013) found that dynein must be complexed with Lis1 and NudE1/NudEL1 to establish appropriate spindle orientations, and later Moon et al. (2014) suggested LIS1 acted via the LIS1-NDEL1-dynein complex to regulate astral MT plus-ends dynamics to establish proper spindle organization. To investigate the possibility of an interaction between NAGK and dynein-Lis1 complex, we conducted NAGK-Lis1 PLA on asynchronous HEK293T cells and then performed tubulin ICC and DAPI counter-staining. As shown in Fig. 3A (arrowheads), NAGK interacted with Lis1 in 

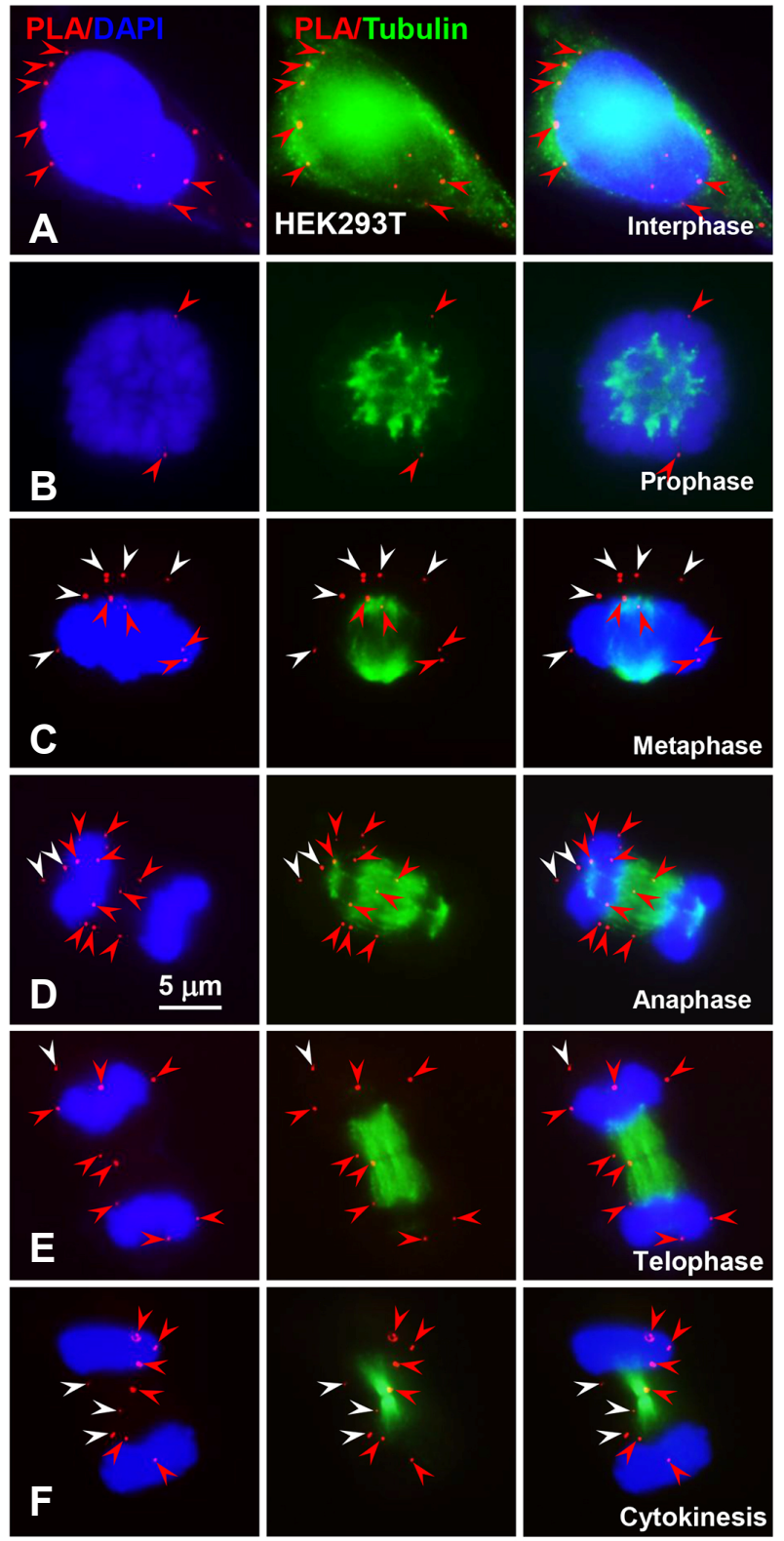

Fig. 2. NAGK-DYNLRB1 interaction during the cell cycle. Proximity ligation assays (PLAs) were performed on HEK293T cells using anti-NAGK and anti-DYNLRB1 antibodies and were followed by ICC using a mouse anti-tubulin antibody (green). Cells were then stained with DAPI to visualize chromosomes. PLA/DAPI, PLA/ICC (tubulin), and merge images are shown. (A) In interphase, NAGK-DYNLRB1 PLA dots around nuclear membrane are indicated by arrowheads. (B) In prophase, chromosomes were condensed (DAPI stained) and NAGKDYNLRB1 PLA signals on nuclear envelopes are shown by arrowheads. (C) In metaphase, PLA signals on chromosomes and/or MT fibers are indicated by red arrowheads and those in cytoplasm are marked with white arrowheads. In the following anaphase (D), telophase (E), and cytokinesis (F) stages, similar distributions of PLA dots were observed. PLA signals on chromosomes and/or MTs are indicated by red arrowheads, and PLA signals in other cytoplasmic areas are indicated by white arrowheads. Scale bar; $5 \mu \mathrm{m}$. cytoplasm and on the outer nuclear membranes in interphase cells, and NAGK-Lis1 complexes were also observed on the nuclear membranes of prophase cells (Fig. 3B; arrowheads). In metaphase, NAGK-Lis1 PLA signals were present in chromosomal alignment and colocalized with MT spindles (Fig. 3C; red arrowheads), and some signals were also observed in astral sites (Fig. 3C; white arrowheads). Similar NAGK-Lis1 interactions were found during anaphase and telophase (Figs. 3D and $3 \mathrm{E})$, on mitotic spindles and on chromosomes (red arrowheads), and in astral cytoplasmic areas (white arrowheads). During cytokinesis, NAGK-Lis1 PLA signals were also observed in chromosomal areas (Fig. 3F; red arrowheads) and in other cytoplasmic areas (Fig. 3F; white arrowheads). Based on these observations, the localization of NAGK-Lis1 interactions (PLA signals) and of NAGK-dynein complexes during cell division were notably similar, particularly in prophase and metaphase cells, which suggested NAGK interacts with dynein-Lis1 complex.

NAGK interacted with dynein-Lis1-NudE1 complex on nuclear envelopes during prophase nuclear invagination

Salina et al. (2002) reported that dynein facilitates prophase nuclear envelope invagination leading to NEB by pulling nuclear membranes. To investigate NAGK-dynein interactions during prophase NEB, we performed NAGK-DYNLRB1 PLA followed by lamin-B ICC and DAPI counter-staining in prophase hypothalamic GT1-7 cells (Fig. 4A) (this cell line was utilized to support our previous findings in HEK293T cells; Fig. 2B). Here, NAGK-dynein PLA signals were also observed around prophase nuclear envelopes (Fig. 4A, arrowheads) and invagination was marked by stronger in situ lamin B signals (Fig. 4A, green arrow) as nuclear lamina disassembles and concentrates at invagination areas during the initiation of prophase NEB (Beaudouin et al., 2002; Georgatos et al., 1997). It has been established that the recruitment or targeting of dynein to nuclear envelopes are regulated by Lis1 (Egan et al., 2012; Splinter et al., 2012) and NudE1/NudEL1 (Bolhy et al., 2011), and dyneinLis1-NudE1 interactions contribute to dynein-dependent nuclear envelope breakdown (Hebbar et al., 2008). We next examined NAGK's role in NEB by studying its interaction with dyneinLis1-NudE1 complex. First, we conducted NAGK-DYNLRB1 PLA followed by Lis1 ICC and DAPI counter-staining in HEK293T cells, and found NAGK-dynein complex (Fig. 4B, original image, inset, red arrowheads) colocalized with Lis1 ICC puncta (Fig. 4B, original image, inset, green arrowheads) at the nuclear envelope during prophase. In a similar experiment on HEK293T cells, NAGK-DYNLRB1 PLA was followed by NudE1 ICC and DAPI counter-staining, and NAGK-dynein-NudE1 interactions were observed on nuclear envelopes during NEB. Furthermore, NAGK-dynein interactions (Fig. 4C, original image, inset, red arrowheads) colocalized with these NudE1 signals (Fig. 4C, original image, inset, green arrowheads). These observations strongly indicate that NAGK interacts with dyneinLis1-NudE1 complex and is involved in prophase NEB.

Colocalization of NAGK with Lis1 and with NudE1 complex on NEs observed after microtubule disruption

Nocodazole, a MT disrupting agent, delays NEB (Salina et al., 2002) and increases Lis1-NudE1/NudEL1-dynein complex accumulation at nuclear envelopes (Hebbar et al., 2008). We reasoned that nocodazole would disrupt both MT and MTassociated NAGK signals but would not change NAGK expression at nuclear envelopes if it interacts with Lis1-NudE1 during NEB. To investigate this rationale, we treated HEK293T cells 


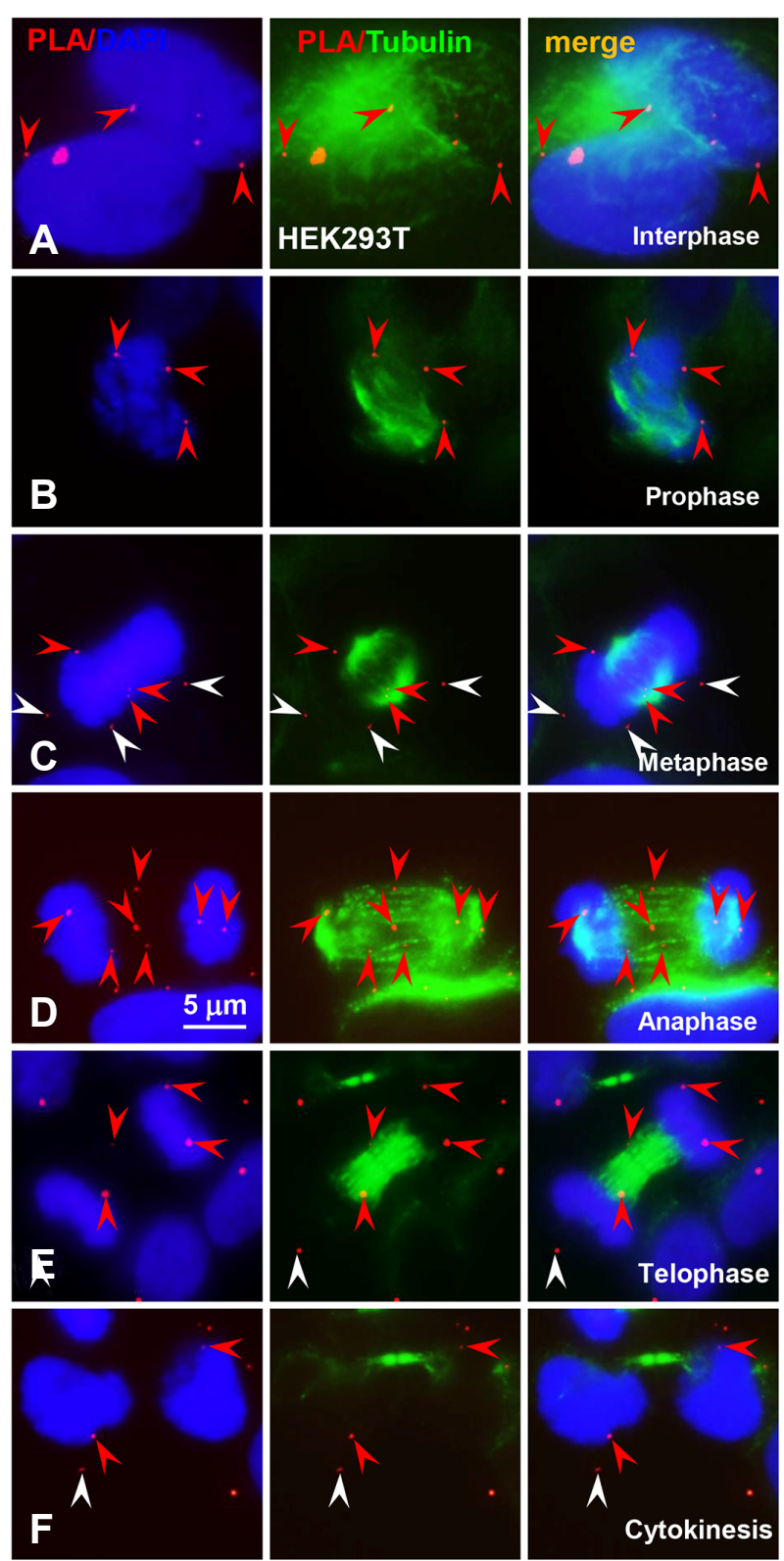

Fig. 3. NAGK-Lis1 interaction during the cell cycle. PLA was carried out on fixed HEK293T cells using primary antibodies against NAGK and Lis1, subsequent ICC was performed using anti-tubulin antibody and then DAPI staining was done. In interphase (A) and prophase cells (B), NAGK-Lis1 interactions were found on nuclear membranes (arrowheads). In metaphase (C), anaphase (D), telophase $(E)$ and cytokinesis $(F)$ stages, PLA signals were observed on chromosomes and/or on MTs (red arrowheads), and in other cytoplasmic areas (white arrowheads). Scale bar; $5 \mu \mathrm{m}$.

with nocodazole to arrest cell division at G2/M transition, at which NEB is a key prerequisite for commitment to $M$ phase. After nocodazole treatment, the proportion of prophase cells increased, and when we double-stained nocodazole treated cells with anti-NAGK and -tubulin antibodies, it was found MTs were disrupted and tubulin staining was mostly present at one
A
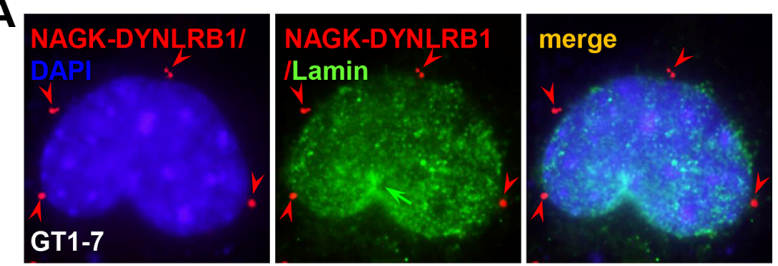

B
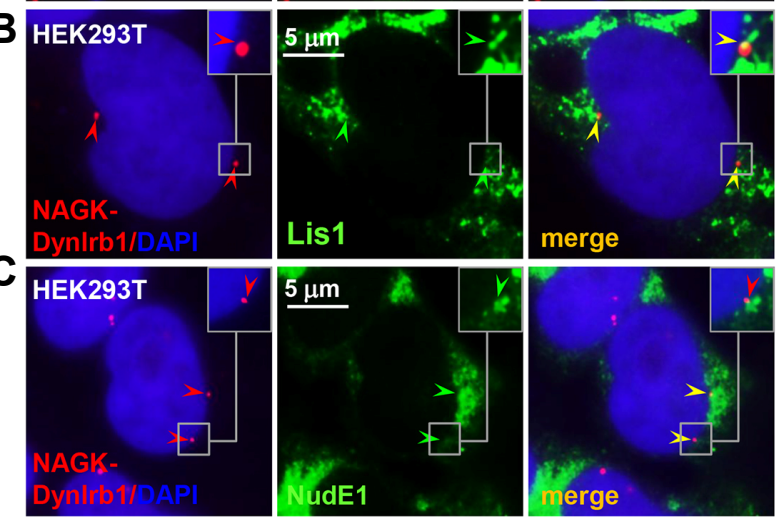

Fig. 4. Interaction between NAGK and DYNLRB1-Lis1-NudE1 complex on nuclear envelopes during prophase nuclear envelope invagination (PNEI). (A) NAGK-DYNLRB-1 PLA and subsequent anti-lamin ICC was conducted on GT1-7 cells, which were then stained with DAPI. PLA signals were observed on nuclear envelopes (arrowheads) during nuclear envelope invagination which is indicated by concentrated lamin staining (green arrow). (B) NAGKDYNLRB1 PLA (red dots), Lis1 ICC (green) and DAPI staining (blue) was performed on fixed HEK293T cells. PLA signals at nuclear envelopes colocalized with Lis1 ICC puncta are shown (original image and inset; arrowheads). (C) NAGK-DYNLRB1 PLA, NudE1 ICC and DAPI staining were performed and colocalizations of NAGK-dynein interactions (red) with NudE1 (green) are shown on nuclear envelopes during PNEI (original image, and inset, arrowheads). Scale bar; $5 \mu \mathrm{m}$.

location, which was assumed to be the centrosome (Fig. 5A, red arrowhead). NAGK signals were also concentrated at this location and overlapped those of tubulin (Fig. 5A, green arrow heads). Furthermore, double-staining in the same manner showed NAGK expression around the nuclear envelope and a significant number of NAGK/Lis1 (Fig. 5B, inset, arrowheads) and NAGK/NudE1 colocalization (Fig. 5C, inset, arrowheads). In addition, cytoplasmic signals for NAGK and Lis1 were weaker than the dispersed cytoplasmic signals observed in the absence of nocodazole (Fig. 5D, arrows). These findings confirm interaction between NAGK and dynein-Lis1-NudE1 complex on nuclear envelopes during the G2/M phase and suggest the participation of NAGK in dynein-mediated NEB.

NAGK interacted with dynein-Lis1-NudE1 complex on chromosomes during metaphase

When we analyzed the positioning of NAGK-dynein (Fig. 6A) and NAGK-Lis1 (Fig. 6B) PLA signals in metaphase cells, both were observed on MT spindles and chromosomes (red arrowheads), suggesting their involvements in chromosome movement and pole focusing, and in other cytoplasmic areas assumed to be related in movement of astral microtubules towards the cortex. Interestingly, majority of NAGK-dynein and 


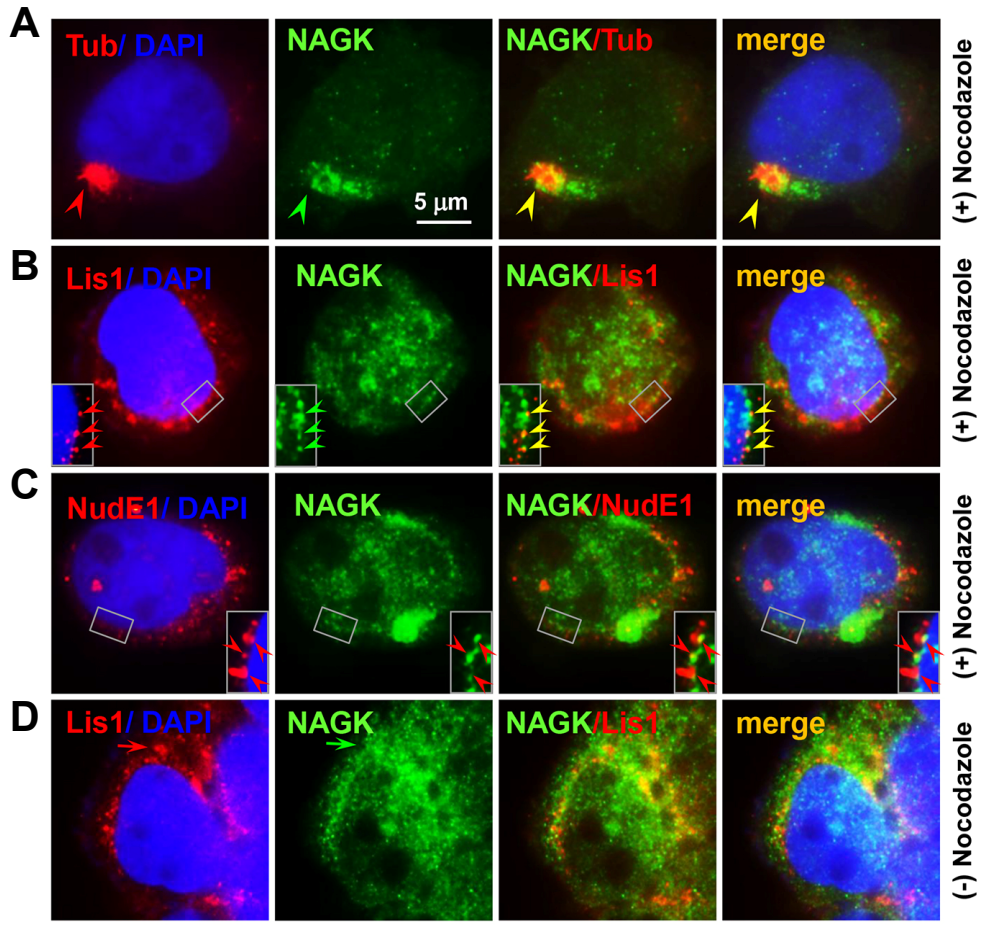

A

a

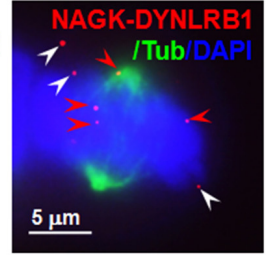

B

a

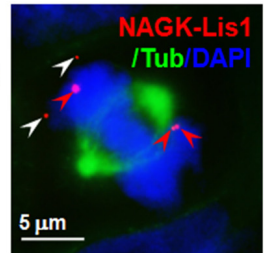

C
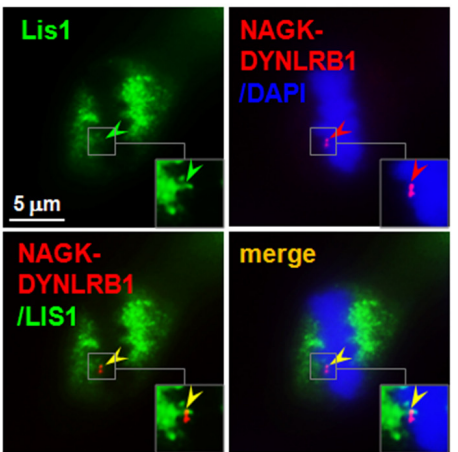

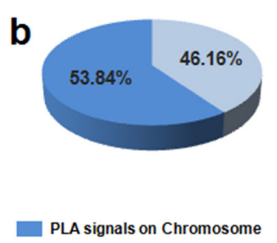

Other PLA signals

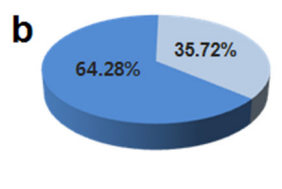

D

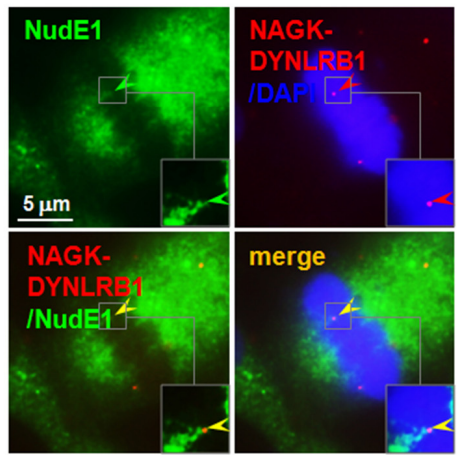

Fig. 5. Colocalization of NAGK with Lis1 and NudE1 on nuclear envelopes after microtubule disruption in prophase cells. HEK293T cells were treated with nocodazole, double labeled with indicated antibodies and then stained with DAPI. (A) Tubulin signals disrupted by nocodazole and concentrated around centrosome are shown by red arrowheads (Tub/DAPI). In addition, colocalization of NAGK signal clusters (green arrowheads, NAGK) with tubulin are indicated by yellow arrowheads (merge). (B) ICCs with antiNAGK (green) and anti-Lis1 (red) primary antibodies were performed on nocodazole treated prophase HEK293T cells, which were then stained with DAPI (blue). DAPI staining revealed invagination of prophase nuclear envelopes. Colocalizations of NAGK signals with Lis1 signals on nuclear envelopes are shown by arrowheads (boxed area enlarged inset) during nuclear invagination. (C) Nocodazole treated prophase HEK293T cells were double stained with anti-NAGK (green) and anti-NudE1 (red) antibodies in a similar method. Colocalization between NAGK and NudE1 signals are shown by arrowheads on a nuclear envelope (arrowheads, inset). (D) ICC doublelabeling with anti-NAGK (green) and anti-Lis1 (red) primary antibodies of control (not treated with nocodazole) HEK293T cells showed dispersed cytoplasmic signals (arrows). Scale bar; $5 \mu \mathrm{m}$.

Fig. 6. NAGK interacted with dynein-Lis1-NudE1 complex on chromosomes in metaphase. (A) (a) NAGK-DYNLRB1 PLA and subsequent tubulin ICC and DAPI staining were performed in HEK293T cells. NAGK-dynein interactions (PLA) on chromosomes are indicated by red arrowheads, and in other cytoplasmic areas by white arrowheads. (b) Statistics: Majority of NAGK-dynein PLA signals were on chromosomes $(53.84 \%)$ in metaphase. (B) (a) NAGK-Lis1 PLA was performed on HEK293T cells and followed by tubulin ICC and DAPI staining. NAGK-Lis1 interactions (PLA) were localized to chromosomes (red arrowheads) and in other cytoplasmic areas (white arrowheads). (b) NAGK-Lis1 interactions in metaphase were occurred on chromosomes $(64.28 \%)$ at a higher frequency compared to other cytoplasmic areas. NAGK-DYNLRB1 PLA followed by Lis1 (C) or NudE1 (D) immunostaining, and DAPI staining revealed colocalizations between NAGK-dynein complex (original image, inset, red arrowheads) and Lis1 (C, original image, inset, green arrowheads), and NudE1 ( $\mathrm{D}$, original image, inset, green arrowheads) in metaphase. Merged images show colocalization on chromosomes (C, D, original image, inset, yellow arrowheads). Scale bar; $5 \mu \mathrm{m}$.
NAGK-Lis1 PLA signals (Figs. 6A-b, 53.84\% and Figs. 6B-b, $64.28 \%$, respectively) were in chromosomal areas. These find- ings imply that NAGK works with dynein and Lis1 to move chromosomes in metaphase cells. Indeed, NAGK-DYNLRB1 

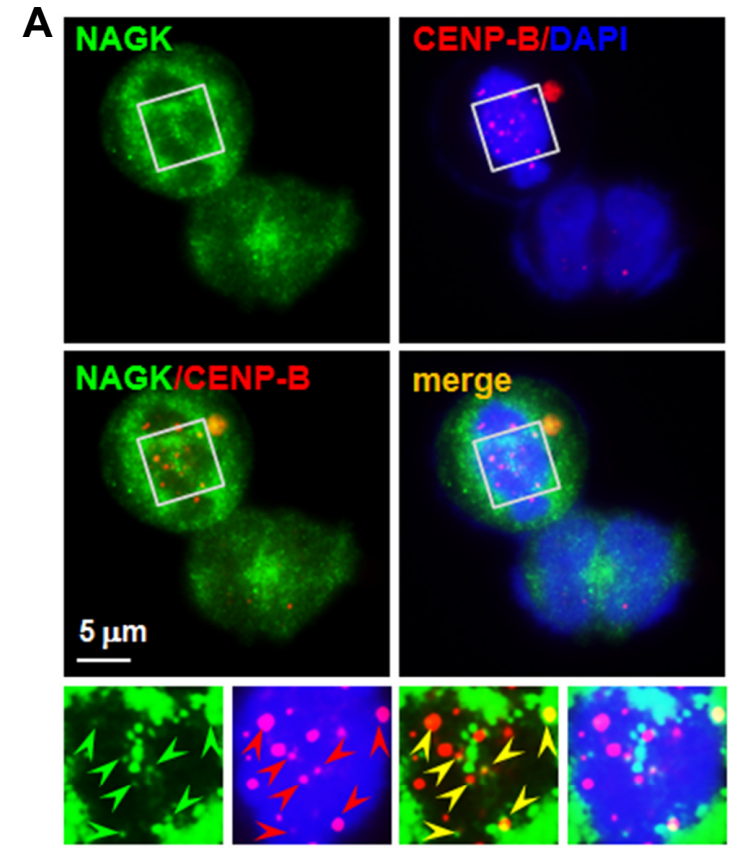

B
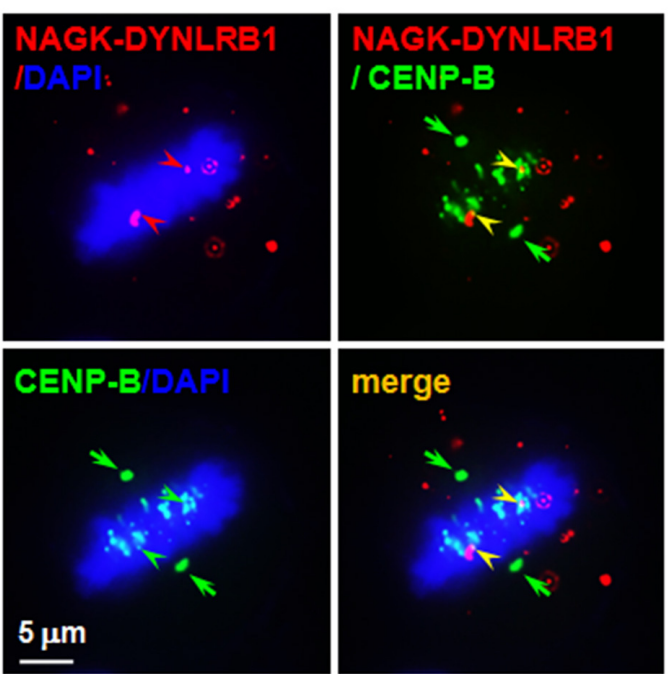

Fig. 7. NAGK-dynein interactions occurred at kinetochores (KTs) in metaphase. (A) NAGK and CENP-B (a marker of kinetochores) double-labeling shows NAGK expression at KTs in HEK293T cells. Boxed areas (enlarged in insets) show colocalization of NAGK signals (green arrowheads) with KTs (red arrowheads, CENP-B) on chromosomes (DAPI, blue) in metaphase. Scale bar; $5 \mu \mathrm{m}$. (B) PLA using anti-NAGK and anti-dynein intermediate chain antibodies was followed by CENP-B ICC and DAPI staining on fixed HEK293T cells and NAGK-dynein complexes (red arrowheads) were found to be linked with CENP-B signals (green arrowheads) at KTs. The localization of NAGK-dynein complexs on KTs are indicated by yellow arrowheads in merged images. Centrosomes were revealed by CENP-B signals (green arrows). Scale bar; $5 \mu \mathrm{m}$.

PLA followed by Lis1 or NudE1 immunostaining and DAPI counterstaining for chromosomes showed that NAGK-dynein complex colocalized with Lis1 (Fig. 6C, original image, inset, arrowheads) and NudE1 (Fig. 6D, original image, inset, arrowheads). These results show that NAGK colocalizes with DYNLRB1-Lis1NudE1 complex on chromosomes in metaphase cells.

Presence of NAGK-dynein complex at kinetochores during metaphase

Kinetochores (KTs) connect chromosomes to the spindle apparatus and pull chromosomes along spindles (Meraldi et al., 2006; Musacchio and Salmon, 2007). It has been reported that Lis1 and NudE1/NudEL1 link dynein to KTs (Liang et al., 2007; Mesngon et al., 2006; Stehman et al., 2007; Vergnolle and Taylor, 2007). To study the presence of NAGK on KTs, we double-stained HEK293T cells with antibodies against NAGK and KT marker protein CENP-B, and found NAGK colocalized with CENP-B at KTs in metaphase cells (Fig. 7A, inset, arrowheads) NAGK-dynein PLA followed by CENP-B immunostaining and DAPI counter-staining was then used to locate NAGK-dynein complex on KTs (Fig. 7B). NAGK-dynein complex (red arrowheads) colocalized with CENP-B (green arrowheads) on chromosomes (DAPI, blue) in metaphase. In addition, CENP-B signals were observed on centrosomes (green arrows). These results confirm that NAGK and dynein interact at KTs in metaphase.

\section{Knockdown of NAGK delayed cell division}

Because the above experiments showed interactions between NAGK and dynein-Lis1-NudE1 complex during different stages of mitotic cell division, we investigated the role of NAGK in cell division. NAGK was knocked-down by transfecting HEK293T cells with a plasmid construct expressing NAGK small hairpin (sh) RNA. Cells were transfected with a red fluorescent protein tagged pDsRed2 control vector (Fig. 8A-a, pDsRed2) or co-transfected with this control vector and NAGK shRNA vector (Lee et al., 2014a) (Fig. 8A-b, sh-NAGK $+\mathrm{pDsRed} 2$ ). After incubation for $48 \mathrm{~h}$ to allow protein expression, live images of transfected cells were captured, and same culture areas were imaged $24 \mathrm{~h}$ later. During this $24 \mathrm{~h}$ (between $48 \mathrm{~h}$ and $72 \mathrm{~h}$ in culture), most cells transfected with control pDsRed2 plasmid divided (Fig. 8A-a, red arrows show the same mark in two different time-lapse images). In contrast, a number of cells transfected with NAGK shRNA vector did not divide (Fig. 8A-b, arrow and arrowheads indicating corresponding cells). Statistical analysis (Fig. 8B, $n=500$ cells) showed during this $24 \mathrm{hr}$ period, control cells multiplied at the same rate as non-transfected cells, and that the transfected to non-transfected cell ratio remained almost the same after 72 $\mathrm{h}$ in culture (Fig. 8B-a). On the other hand cells transfected with NAGK shRNA vector did not divide at the normal rate as evidenced by a decline in transfected to total cell ratio (Fig. $8 \mathrm{~B}-\mathrm{b})$. These results show that NAGK knockdown delayed cell division process because of lack of interaction between NAGK and dynein-Lis1-NudE1 complex.

\section{DISCUSSION}

This study was undertaken to investigate the role of NAGKdynein complexes during cell division. The eukaryotic cell cycle is primarily divided into two basic phases, that is, mitosis and interphase. Interphase consists of three different phases: two gap phases (G1 and $\mathrm{G} 2$ ) and an $\mathrm{S}$ (synthesis) phase, during which genetic material is duplicated. In the G2 phase, cells assemble the cytoplasmic materials necessary for mitosis (the $\mathrm{M}$ phase), a continuous process comprised of five different stages: prophase, prometaphase, metaphase, anaphase, and 
A

a

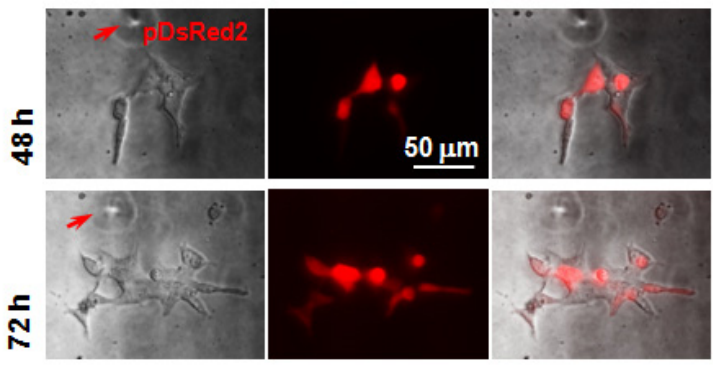

b

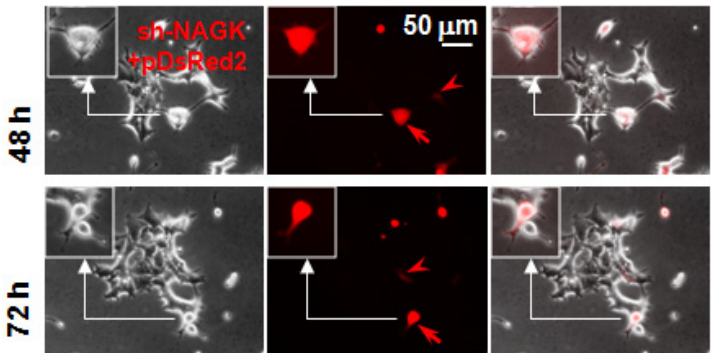

B

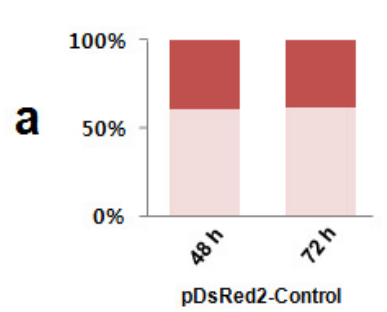

(\%) Transfected cells (\%) Non-transfected cells

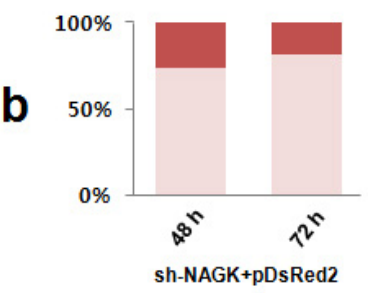

Fig. 8. Knockdown of NAGK caused a delay in cell division. HEK293T cells were transfected with indicated plasmids and images of same culture areas were captured after 48 and $72 \mathrm{~h}$. In epifluorescent live cell images, transfected cells appeared bright red. (a) Most cells transfected with control (pDsRed2) vector plasmid divided after incubation from $48 \mathrm{~h}$ to $72 \mathrm{~h}$ after transfection. Specific culture areas are marked with red arrows to show the same region photographed in timelapse images. (b) Cells co-transfected with pDsRed2 vector and NAGK shRNA plasmid did not divide between $48 \mathrm{~h}$ and $72 \mathrm{~h}$. Two transfected cells are indicated by arrow and arrowheads, respectively in time-lapse images. Inset shows one representative cell that did not divide at $24 \mathrm{~h}$. Scale bar; $50 \mu \mathrm{m}$. (B) Transfected and nontransfected cells were counted after 48

$\mathrm{h}$ and $72 \mathrm{~h}$ incubation in the same areas and plotted $(\mathrm{n}=500)$. (a) Cells transfected with pDsRed2 control vector plasmid divided at approximately the same transfected to non-transfected cell ratio. (b) Cells co-transfected with NAGK shRNA and pDsRed2 vector did not divide as quickly as non-transfected cells and the transfected to non-transfected cell ratios declined.

telophase. Prophase is the initial phase during which NEB, centrosome duplication, and mitotic spindle formation take place. Later, daughter chromosomes separate during the metaphase, anaphase, and telophase stages, to finally produce two daughter cells by cytokinesis (Lodish et al., 2000). During cell division, minus-end directed molecular motor dynein interacts with different adaptor proteins like Lis1, NudE1/NudEL1 to play different roles during centrosome separation, chromosome movements, spindle organization, spindle positioning, and mitotic checkpoint silencing (Raaijmakers and Medema, 2014). At the very beginning of mitosis, dynein localized on the nuclear envelope contributes to NEB (Salina et al., 2002). We previously reported ring-like NAGK expression on the cytoplasmic faces of outer nuclear membranes (Sharif et al., 2015) and that NAGK interacts with DYNLRB1 (Islam et al., 2015). In the present study, a similar distribution of NAGK-dynein complexes were observed around the outer nuclear membrane during prophase NEB initiation, and furthermore, these complexes were found to be colocalized with Lis1 and NudE1. In addition, we observed NAGK-Lis1 interaction on prophase nuclear envelopes. Mechanical shearing of nuclear membrane is a critical step during NEB and is preceded by prophase nuclear envelope invaginations (PNEls) near newly duplicated centrosomes. It has been reported PNEI formation is dynein-dependent (Salina et al., 2002) and that both Lis1 and NudE1/NudEL1 influence PNEI in cultured cell lines and in the developing mouse brain (Hebbar et al., 2008). Thus, the NAGK-Lis1 complexes and the colocalization of NAGK-dynein complexes with Lis1 and NudE1 on prophase nuclear envelopes suggest that NAGK plays a role during prophase NEB by interacting with dynein-Lis1-NudE1 complexes (Fig. 9A).

Mitotic cell division starts with NEB driven by MT-induced deformation. Nocodazole disrupts MT fibers and delays prophase NEB, which results in an accumulation of Lis1-NudE1/NudEL1- dynein complex at the prophase nuclear envelope (Cockell et al., 2004; Coquelle et al., 2002; Hebbar et al., 2008; Smith et al., 2000). In the present study, MT associated NAGK expression collapsed in HEK293T cells treated with nocodazole and concentrated around centrosome and on nuclear envelope. Colocalization of latter NAGK punctae with Lis1 and NudE1 on nuclear envelopes occurred, confirming an interaction between NAGK and dynein-Lis1-NudE1 complex during prophase NEB. In addition, we found NAGK-dynein complexes interacted with Lis1 and NudE1 on metaphase chromosomes, and that NAGK or NAGK-dynein complex colocalized with CENP-B (a KT marker protein) in metaphase chromosomes. Dynein links tubulin to kinetochores (KTs) and adopts a force generating role during metaphase. Dynein is recruited to $\mathrm{KTs}$ during (pro)metaphase in two distinct ways. The first involves RZZ (Rod, Zwilch, and Zw10) complex in KTs, which recruits dynein complex (Karess, 2005) via an interaction between Zw10 and the p50 subunit of dynactin (Starr et al., 1998). The second involves CENP-F, Lis1, and NudE1/NudEL1 (Vergnolle and Taylor, 2007) whereby Lis1 and NudE1/NudEL1 work together with dynein complex to link MT fibers to KTs (Liang et al., 2007; Mesngon et al., 2006; Stehman et al., 2007). In addition, Raaijmakers et al. (2013) found that dynein intermediate chain 2, DYNLRB1 and TCTEX1/1L all contribute to recruitment of the dynein complex to the KTs. Therefore, our observation of an interaction between NAGK and dynein-Lis1-NudE1/NudEL1 complex at metaphase KTs suggests that NAGK plays a role in the dynein-mediated connection of MTs and KTs (Fig. 9B).

In the present study, NAGK-dynein and NAGK-Lis1 complexes were observed at the astral part of the mitotic spindles. Human mitotic spindle consists of different types of microtubules: those that emanates from centrosomes to interact with KTs are known as K-fibers; those that connect to cell cortex are termed astral MTs, and those that form antiparallel overlap between opposing 


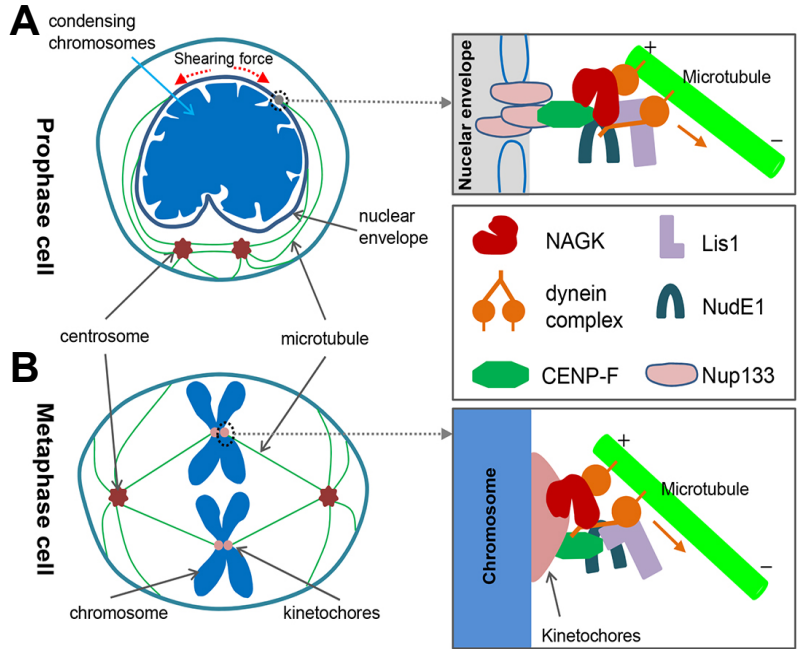

Fig. 9. Diagram representing the interaction between NAGK, dynein, and the dynein adaptor proteins Lis1 and NudE1 in prophase and metaphase. (A) Prophase. In cells at prophase, centrosomes duplicate and MTs connect to nuclear envelope proteins, like Nup 133, with the aid of dynein complex and adaptor proteins, such as, Lis1, NudE1, and CENP-F (Bolhy et al., 2011, Hebbar et al., 2008, Vergnolle and Taylor, 2007). Chromosomes are in a condensing state and centrosome-directed force (two red dotted arrows) generated by dynein movement creates nuclear envelope invagination in areas close to centrosomes, and causes breakdown in the nuclear envelope distal to centrosomes. We propose by binding to dynein and nuclear envelope, NAGK works as an adaptor to anchor dynein complex to the nuclear envelope and regulates its movement along MTs. (B) Metaphase. Centrosomes have moved to opposite ends of the cell, chromosomes align in the equatorial plane, and MT spindle fibers connect to KTs with the aid of dynein and its adaptor proteins. Our findings indicate that NAGK acts as an adaptor protein in the recruitment of dynein to KTs.

centrosomes are called polar MTs. Lis1 directs dynein to the plus end on astral MTs and prevents it walking toward the minus end by keeping it in an 'off' state (Markus and Lee, 2011). Lis1 reduces dynein motility, and thus, maintains dynein in a MT-bound state at the plus end, which is considered the 'off' state for minus end directed dynein motor complex. The presence of NAGK-dynein and NAGK-Lis1 complexes at astral site of MT spindle suggests that NAGK might also function with dynein-Lis1 complex at the cell cortex.

In the present study, NAGK knockdown delayed cell division, and we conjecture that since NAGK regulates dynein motor complex, which plays a critical role in cell division, the observed delay in cell cycle could have been due to a malfunction of dynein during mitosis. Raaijmakers et al. (2013) undertook a systemic investigation of dynein regulators using siRNAs targeting individual subunits of dynein and its adaptors, such as, dynactin, Lis1, and NudE1/NudEL1, and reported that depletion of DYNLRB1, Lis1, or NudE1/NudEL1 led to severe centrosome detachment in prophase, severely reduced dynein-KT attachment, and led to a dramatic defect in chromosome alignment, which ultimately increased mitotic index, that is, the ratio of the number of cells undergoing mitosis to the total number of cells. In the present study, knockdown of NAGK by shRNA delayed the cell cycle by more than $24 \mathrm{~h}$, and it has been previously reported native dynein requires the presence of many adaptors for the correct localization and activation of the dynein complex during mitosis, for example, dynein regulators, such as, Lis1 and NudE1/NudEL1, are required to generate sufficient force (Raaijmakers et al., 2013; Tanenbaum et al., 2008). For spindle pole focusing, the p150Glued subunit of dynactin binds with MTs (Waterman-Storer et al., 1995). Furthermore, Lis1, NudE1/NudEL1, numerous dynein-binding adaptor proteins like ZW10 (Starr et al., 1998; Whyte et al., 2008), hSpindly (Gassmann et al., 2008; 2010; Griffis et al., 2007), NuMA (Fant et al., 2004), CENP-F (Vergnolle and Taylor, 2007) as well as dynactin, contribute to the targeting of dynein complex to KTs. In addition, dynein is known to be recruited to the nuclear envelope during G2/prophase in a BICD2-dependent manner (Raaijmakers et al., 2012; Splinter et al., 2010). In view of the many adaptors of dynein required during mitosis, we propose NAGK might be another important adaptor that enables dynein complex to function efficiently, especially during prophase NEB and metaphase chromosome seperation. Further molecular dissection of the roles of NAGK-binding complexes in mitosis is required.

\section{ACKNOWLEDGMENTS}

This research was supported by Basic Science Research Program through the National Research Foundation of Korea (NRF) funded by the Ministry of Science, ICT and Future Planning to ISM (2015R1A2A2A01007104).

\section{REFERENCES}

Beaudouin, J., Gerlich, D., Daigle, N., Eils, R., and Ellenberg, J. (2002). Nuclear envelope breakdown proceeds by microtubuleinduced tearing of the lamina. Cell 108, 83-96.

Bolhy, S., Bouhlel, I., Dultz, E., Nayak, T., Zuccolo, M., Gatti, X., Vallee, R., Ellenberg, J., and Doye, V. (2011). A Nup133dependent NPC-anchored network tethers centrosomes to the nuclear envelope in prophase. J. Cell Biol. 192, 855-871.

Cockell, M.M., Baumer, K., and Gonczy, P. (2004). Lis-1 is required for dynein-dependent cell division processes in C. elegans embryos. J. Cell Sci. 117, 4571-4582.

Coquelle, F.M., Caspi, M., Cordelières, F.P., Dompierre, J.P., Dujardin, D.L., Koifman, C., Martin, P., Hoogenraad, C.C., Akhmanova, A., Galjart, N., et al. (2002). LIS1, CLIP-170's key to the dynein/dynactin pathway. Mol. Cell. Biol. 22, 3089-3102.

Datta, A. (1970). Studies on hog spleen $\mathrm{N}$-acetylglucosamine kinase. I. Purification and properties of $\mathrm{N}$-acetylglucosamine kinase. Biochim. Biophys. Acta 220, 51-60.

Dujardin, D.L., and Vallee, R.B. (2002). Dynein at the cortex. Curr. Opin. Cell Biol. 14, 44-49.

Egan, M.J., Tan, K., and Reck-Peterson, S.L. (2012). Lis1 is an initiation factor for dynein-driven organelle transport. J. Cell Biol. 197, 971-982.

Esko, J.D., and Lindahl, U. (2001). Molecular diversity of heparin sulfate. J. Clin. Invest. 108, 169-173.

Fant, X., Merdes, A., and Haren, L. (2004). Cell and molecular biology of spindle poles and NuMA. Int. Rev. Cytol. 238, 1-57.

Gassmann, R., Essex, A., Hu, J.S., Maddox, P.S., Motegi, F., Sugimoto, A., O'Rourke, S.M., Bowerman, B., McLeod, I., Yates, J.R. III., et al. (2008). A new mechanism controlling kinetochoremicrotubule interactions revealed by comparison of two dyneintargeting components: SPDL-1 and the Rod/Zwilch/Zw10 complex. Genes Dev. 22, 2385-2399.

Gassmann, R., Holland, A.J., Varma, D., Wan, X., Civril, F., Cleveland, D.W., Oegema, K., Salmon, E.D., and Desai, A. (2010). Removal of Spindly from microtubule-attached kinetochores controls spindle checkpoint silencing in human cells. Genes Dev. 2, 957-971.

Georgatos, S.D., Pyrpasopoulou, A., and Theodoropoulos, P.A. (1997). Nuclear envelope breakdown in mammalian cells involves stepwise lamina disassembly and microtubule-drive deformation of the nuclear membrane. J. Cell Sci. 110, 2129-2140.

Griffis, E.R., Stuurman, N., and Vale, R.D. (2007). Spindly, a novel 
protein essential for silencing the spindle assembly checkpoint, recruits dynein to the kinetochore. J. Cell Biol. 177, 1005-1015.

Hakomori, S. ( 2000). Traveling for the glycosphingolipid path. Glycoconj. J. 17, 627-647.

Hebbar, S., Mesngon, M.T., Guillotte, A.M., Desai, B., Ayala. R., and Smith, D.S. (2008). Lis1 and Ndel1 influence the timing of nuclear envelope breakdown in neural stem cells. J. Cell Biol. 182, 1063-1071.

Hinderlich, S., Berger, M., Schwarzkopf, M., Effertz, K., and Reutter, W. (2000). Molecular cloning and characterization of murine and human $N$-acetylglucosamine kinase. Eur. J. Biochem. 267, 33013308.

Howell, B.J., McEwen, B.F., Canman, J.C., Hoffman, D.B., Farrar E.M., Rieder, C.L., and Salmon, E.D. (2001). Cytoplasmic dynein/dynactin drives kinetochore protein transport to the spindle poles and has a role in mitotic spindle checkpoint inactivation. J. Cell Biol. 155, 1159-1172.

Hu, D.J.-K., Baffet, A.D., Nayak, T., Akhmanova, A., Doye, V., and Vallee, R.B. (2013). Dynein recruitment to nuclear pores activates apical nuclear migration and mitotic entry in brain progenitor cells. Cell 154, 1300-1313.

Hurley, J.H. (1996). The sugar kinase/heat shock protein 70/actin superfamily: implications of conserved structure for mechanism. Annu. Rev. Biophys. Biomol. Struct. 25, 137-162.

Islam, M.A., Sharif, S.R., Lee, H.S., Seog, D.H., and Moon, I.S. (2015a). N-acetyl- $D$-glucosamine kinase interacts with dynein light chain roadblock type 1 at Golgi outposts in neuronal dendritic branch points. Exp. Mol. Med. 47, e177.

Islam, M.A., Sharif, S.R., Lee, H.S., and Moon, I.S. (2015b). Nacetyl- $D$-glucosamine kinase promotes the axonal growth of developing neurons. Mol. Cells 38, 876-885.

Karess, R. (2005). Rod-Zw10-Zwilch: a key player in the spindle checkpoint. Trends Cell Biol. 15, 386-392.

Kiyomitsu, T., and Cheeseman, I.M. (2012). Chromosome- and spindle-pole derived signals generate an intrinsic code for spindle position and orientation. Nat. Cell Biol. 14, 311-317.

Lee, H.S., Cho, S.J., and Moon, I.S. (2014a). The non-canonical effect of $N$-acetyl- $D$-glucosamine kinase on the formation of neuronal dendrites. Mol. Cells 37, 248-256.

Lee, H.S., Dutta, S., and Moon, I.S. (2014b). Upregulation of dendritic arborization by $N$-acetyl- $D$-glucosamine kinase is not dependent on its kinase activity. Mol. Cells 37, 322-329.

Liang, Y., Yu, W., Li, Y., Yu, L., Zhang, Q., Wang, F., Yang, Z., Du, J. Huang, Q., Yao, X., et al. (2007). Nudel modulates kinetochore association and function of cytoplasmic dynein in $\mathrm{M}$ phase. Mol. Biol. Cell 18, 2656-2666.

Ligos, J.M., de Lera, T.L., Hinderlich, S., Guinea, B., Sanchez, L., Roca, R., Valencia, A., and Bernad, A. (2002). Functional interaction between the Ser/Thr kinase PKL12 and Nacetylglucosamine kinase, a prominent enzyme implicated in the salvage pathway for GlcNAc recycling. J. Biol. Chem. 277, 63336343.

Lodish, H., Berk, A., Zipursky, S.L., Matsudaira, P., Baltimore, D., and Darnell, J. (2000). Overview of the Cell Cycle and Its Control. In Molecular Cell Biology, 4th eds. (New York: W. H. Freeman), Section 13.1.

Markus, S.M., and Lee, W.L. (2011). Microtubule-dependent path to the cell cortex for cytoplasmic dynein in mitotic spindle orientation. Bioarchitecture 1, 209-215.

Meraldi, P., McAinsh, A.D., Rheinbay, E., and Sorger, P.K. (2006). Phylogenetic and structural analysis of centromeric DNA and kinetochore proteins. Genome Biol. 7, R 23

Mesngon, M.T., Tarricone, C., Hebbar, S., Guillotte, A.M., Schmitt, E.W., Lanier, L., Musacchio, A., King, S.J., and Smith, D.S. (2006). Regulation of cytoplasmic dynein ATPase by Lis1. J. Neurosci. 26, 2132-2139.

Moon, I.S., Cho, S.J., Jin, I., and Walikonis, R. (2007). A simple method for combined fluorescence in situ hybridization and immunocytochemistry. Mol. Cells 24, 76-82.

Moon, H.M., Youn, Y.H., Pemble, H., Yingling, J., Wittmann, T., and Wynshaw-Boris, A. (2014). LIS1 controls mitosis and mitotic spindle organization via the LIS1-NDEL1-dynein complex. Hum. Mol. Genet. 23, 449-466.

Musacchio, A., and Salmon, E.D. (2007). The spindle-assembly checkpoint in space and time. Nat. Rev. Mol. Cell Biol. 8, 379393.
Ori-McKenney, K.M., Jan, L.Y., and Jan, Y.N. (2012). Golgi outposts shape dendrite morphology by functioning as sites of acentrosomal microtubule nucleation in neurons. Neuron 76, 921-930.

Pfarr, C.M., Coue, M., Grissom, P.M., Hays, T.S., Porter, M.E., and McIntosh, J.R. (1990). Cytoplasmic dynein is localized to kinetochores during mitosis. Nature 345, 263-265.

Raaijmakers, J.A., and Medema, R.H. (2014). Function and regulation of dynein in mitotic chromosome segregation. Chromosoma $123,407-422$

Raaijmakers, J.A., van Heesbeen, R.G., Meaders, J.L. Geers, E.F. Fernandez-Garcia, B., Medema, R.H., and Tanenbaum, M.E. (2012). Nuclear envelope-associated dynein drives prophase centrosome separation and enables Eg5-independent bipolar spindle formation. EMBO J. 31, 4179-4190.

Raaijmakers, J.A., Tanenbaum, M.E., and Medema, R.H. (2013). Systematic dissection of dynein regulators in mitosis. J. Cell Biol. 201, 201-215

Salina, D., Bodoor, K., Eckley, D.M., Schroer, T.A., Rattner, J.B., and Burke, B. (2002). Cytoplasmic dynein as a facilitator of nuclear envelope breakdown. Cell 108, 97-107.

Schachter, H. (2000). The joys of HexNAc. The synthesis and function of $\mathrm{N}$ - and O-glycan branches. Glycoconj. J. 17, 465-483.

Sharif, S.R., Lee, H.S., Islam, M.A., Seog, D.H., and Moon, I.S. (2015). $N$-acetyl- $D$-glucosamine kinase is a component of nuclear speckles and paraspeckles. Mol. Cells 38, 402-408.

Sharp, D.J., Rogers, G.C., and Scholey, J.M. (2000). Cytoplasmic dynein is required for poleward chromosome movement during mitosis in Drosophila embryos. Nat. Cell Biol. 2, 922-930.

Shu, T., Ayala, R., Nguyen, M.D., Xie, Z., Gleeson, J.G., and Tsai, L.H. (2004). Ndel1 operates in a common pathway with LIS1 and cytoplasmic dynein to regulate cortical neuronal positioning. Neuron 44, 263-277.

Smith, D.S., Niethammer, M., Ayala, R., Zhou, Y., Gambello, M.J., Wynshaw-Boris, A., and. Tsai, L.H. (2000). Regulation of cytoplasmic dynein behaviour and microtubule organization by mammalian Lis1. Nat. Cell Biol. 2, 767-775.

Splinter, D., Tanenbaum, M.E., Lindqvist, A., Jaarsma, D., Flotho, A. Yu, K.L., Grigoriev, I., Engelsma, D., Haasdijk, E.D., Keijzer, N., et al. (2010). Bicaudal D2, dynein and kinesin-1 associate with nuclear pore complexes and regulate centrosome and nuclear positioning during mitotic entry. PLoS Biol. 8, e1000350.

Splinter, D., Razafsky, D.S., Schlager, M.A., Serra-Marques, A., Grigoriev, I., Demmers, J., Keijzer, N., Jiang, K., Poser, I., Hyman, A.A., et al. (2012). BICD2, dynactin, and LIS1 cooperate in regulating dynein recruitment to cellular structures. Mol. Biol. Cell 23, 4226-4241.

Starr, D.A., Williams, B.C., Hays, T.S., and Goldberg, M.L. (1998). ZW10 helps recruit dynactin and dynein to the kinetochore. J. Cell Biol. 142, 763-774.

Stehman, S.A., Chen, Y., McKenney, R.J., and Vallee, R.B. (2007). NudE and NudEL are required for mitotic progression and are involved in dynein recruitment to kinetochores. J. Cell Biol. 178, 583-594.

Steuer, E.R., Wordeman, L., Schroer, T.A., and Sheetz, M.P. (1990). Localization of cytoplasmic dynein to mitotic spindles and kinetochores. Nature 345, 266-268.

Tanenbaum, M.E., Macůrek, L., Galjart, N., and Medema, R.H. (2008). Dynein, Lis1 and CLIP-170 counteract Eg5-dependent centrosome separation during bipolar spindle assembly. EMBO J. 27, 3235-3245

Tanenbaum, M.E., Akhmanova, A., and Medema, R.H. (2010). Dynein at the nuclear envelope. EMBO Rep. 11, 649.

Van den Steen, P., Rudd, P.M., Dwek, R.A., and Opdenakker, G (1998). Concepts and principles of O-linked glycosylation. Crit. Rev. Biochem. Mol. Biol. 33, 151-208.

Vergnolle, M.A., and Taylor, S.S. (2007). Cenp-F links kinetochores to Ndel1/Nde1/Lis1/dynein microtubule motor complexes. Curr. Biol. 17, 1173-1179.

Varma, D., Monzo, P., Stehman, S.A., and Vallee, R.B. (2008). Direct role of dynein motor in stable kinetochore-microtubule attachment, orientation, and alignment. J. Cell Biol. 182, 10451054.

Waterman-Storer, C.M., Karki, S., and Holzbaur, E.L. (1995) The p150Glued component of the dynactin complex binds to both microtubules and the actin-related protein centractin (Arp-1). Proc. Natl. Acad. Sci. USA 92, 1634-1638. 
NAGK Regulates Dynein in Cell Division Syeda Ridita Sharif et al.

Whyte, J., Bader, J.R., Tauhata, S.B., Raycroft, M., Hornick, J., Pfister, K.K., Lane, W.S., Chan, G.K., Hinchcliffe, E.H., Vaughan, P.S., et al. (2008). Phosphorylation regulates targeting of cytoplasmic dynein to kinetochores during mitosis. J. Cell Biol. 183, 819-834.
Yan, X., Li, F., Liang, Y., Shen, Y., Zhao, X., Huang, Q., and Zhu, X. (2003). Human Nudel and NudE as regulators of cytoplasmic dynein in poleward protein transport along the mitotic spindle. Mol. Cell. Biol. 23, 1239-1250. 